\title{
Research on Current Situation, Problems and Countermeasures of Core Strength Training of College Athletes
}

\author{
Wenjie Zhu \\ P.E.Institute of Xuchang University, Xuchang, 461000, China
}

Keywords: College athletes, Core strength training, Core stability

\begin{abstract}
Core area strength is the main factor to determine the strength index of college athletes. Core strength training is a common training method for college athletes. However, there are many problems in the core strength training of Chinese college athletes at the present stage. This paper analyzes these problems and puts forward the countermeasures of upgrading training concept, improving training content and enriching training methods to provide some references for the relevant researchers.
\end{abstract}

\section{Current Situation of Core Strength Training of College Athletes}

The core refers to the interruption of the body's body, which consists of the abdominal muscles, hip muscles, and the back muscles. The core muscle is a muscle that balances the body's balance, ranging from the chest to the middle of the thigh. For decades, experts and scholars have done a lot of research on the strength training, and in real life force in sports training plays an important role, but the study found that the basic strength did not increase to improve the competitive level of athletes, coaches will only focus on more specific strength, not from the core muscle strength point of view. Judging from the special skills of tennis, the core strength is the key to tennis speed and strength, especially for the tennis players of weak overall strength, the training of core strength is imminent. From a biological point of view, we can know that the enhancement of core stability is achieved by strengthening the strength of the core muscles. At present, domestic strength training mainly has seven main training methods, such as weight resistance, resistance exercises, overcoming the practice of elastic objects and overcoming the external environment resistance. The physical fitness of college athletes is one of the basic conditions for deciding the outcome of a competition. And the core strength is a connecting link between the preceding and the next, connecting the upper and lower limbs, and playing a pivotal role. With good core strength, you can better control the stability of your body. Therefore, the development of the core strength directly affects the physical fitness, technical and tactical level and competitive level of the basketball players. It is not difficult to find out that it is not our technical and tactical backwardness, but the bottleneck lies in the backwardness of physical fitness. Without ample physical reserves, there is no strong guarantee for training competitions. This research takes the training of core strength of college students as the breakthrough point, and explores the mode of training special core strength for college athletes.

\section{Problems of Core Strength Training of College Athletes}

Backward Training Concept. The process of strength training is a thorough, accurate, complicated and tedious process, if not with a reasonable training plan, athlete's strength cannot be improved effectively, even caused by sports injuries and influence of sports life. In the early stages of strength training, if the earth strength training resulted in maximum load strength training is close to or more than the body can bear, will destroy the balance of muscle tissue, leading to muscle and ligament tear, resulting in sports injuries. Therefore, to control the muscle strength of the growth rate 
of science, follow the following principles: first, to reduce the risk of injury to do warm-up exercise the muscles ready to work, you can start to stretch, and then in power and load when training a small to large, first from the general load, then should pay attention to the image and keep good action. Fast strength is very important in tennis, the power quality, the absolute majority of people think the power quality is very important, the importance of fast strength and strength quality in tennis training usually has also been fully reflected. But in the core strength training, most of the coaches are not particularly understand, theories and concepts of core strength and understand the coaches are relatively vague, generally believe that the core strength is lumbar muscle strength, the core strength training is equivalent to the lumbar muscle strength training, does not distinguish between core strength and lumbar muscle strength. The stable body core strength maintains the correct body posture, improve body control and balance ability, improve the movement from the core to the limbs and other muscles of the whole muscle energy output. The core muscles in the body for the stability of the center of gravity, link force, transmission power function, but also the main part of the whole force, plays a pivotal role in connecting the upper and lower limb coordination and integration of force. Therefore, the training of the core strength of the waist plays an important role in the whole function of the human body. The core power is the core area of muscle strength, which bears the control center, stable equilibrium, conduction under the power and stable position, is an important foundation for the whole force, activity and stability, on the limbs and trunk force plays a pivotal role.

Unreasonable Training Content. In the core strength training, we should do technical specifications and pay attention to quality improvement. However, there are nonstandard and irrational movement techniques in core strength training. The nonstandard movement technique will lead to poor training effect. For example, training the waist, upper abdominal strength, the team's action range did not meet the technical requirements, disrupted the core of the law, and thus cannot complete the coach to develop detailed weekly plans, monthly plans, and even the entire cycle plan. Core strength, movement difficulty causes psychological barrier. In the core strength training process, because the movement difficulty is big, creates the psychological pressure, finally causes the training effect not to be ideal. In the training process, it is not difficult to find that the athletes who have been exposed to the core strength training have shown a positive attitude in the core strength training. To some extent, be able to do active training. Many athletes who have never been trained in the core have shyness, lack of training courage, leading to a decline in enthusiasm, and ultimately affect the quality of training. During the preparation period, the emphasis should be on the number and frequency of each training phase during the muscle strength training period. The last is the direction of movement, strength training due to the original direction of the body and the opposite direction of muscle contraction, therefore, in strength training must be carried out after the end of relaxation exercise, stretch to avoid sports injuries. The increase in the strength of the small muscle group did not greatly improve the skills of the college students, nor did it change as clearly as the professional college athletes. But on the other hand, the improvement of the core strength can better stabilize the body, control movements, and better grasp the special action of the students.

Single Training Method. Most of the students get athlete's strength is through the gym, multi-function room for conventional strength training load to stimulate in order to enhance the strength of muscles, which is based on conventional strength training, is a general strength training, the main purpose is to lay the foundation for the special strength training. However, it has been proved that although the strength has been improved after regular strength training, it has not much effect on College Students' athletic performance, special strength and competitive level. However, studies have found that after the basic strength training, the special strength training of college students can not only make the muscles become stronger and more coordinated, but also improve the utilization of the basic strength in the competition. Therefore, the training of basic strength should be combined with special technical movements. Found in their daily training, most college athletes and coaches of college students tend to ignore the small muscles and excessive attention to the big muscles, even simply ignore the small muscles of the training, direct strength training of limbs. Therefore, in the process of college students, the trunk has become a regular part of college athletes, and the athlete's trunk is unbalanced. Studies have shown that the strength of abdominal muscles of 
high-level college athletes is far greater than the strength of the dorsal muscles. Domestic strength experts, sports scholars and college coaches have always attached great importance to the training of athletes' abdominal muscle strength, but still do not avoid attention to large muscle mass, ignoring the phenomenon of small muscle mass. Therefore, we should strength training in the coordinated development of the overall strength and local strength, according to the actual situation of teaching, according to local conditions, so that the overall strength of college athletes improve coordination ability, effectively prevent sports injury unnecessary.

\section{Countermeasures of Core Strength Training of College Athletes}

Upgrade Training Concept. Core strength training is based on the kinematic chain theory in completing the technical action process. It will participate in the completion of the action of the body into a chain, each part of the body movement is a part of the chain, the completion of the action is to rely on technology to achieve the transfer of momentum in each link between the core strength is in the momentum transfer process in the power chain play a key role transfer the effective transmission of upper limbs plays a pivotal role in the stability of the core can be strong and stable from the ground forces in the process of the momentum of the upper and lower limbs, in order to achieve the maximum acceleration of the upper limb or instruments or deceleration, can also be upper momentum transfer to the lower extremity, lower extremity muscle strength adjustment effect to the ground. In order to improve the upper and lower limbs or action coordination between the work efficiency. The combination structure of basketball techniques is complex, and the tactics are well integrated. At the same time, the speed of attack and defense is emphasized. Good core strength can provide a fulcrum and stability for the rapid movement of the body, or even to a certain extent, affect the quality of the body in the air and all kinds of technical movements, to jump stop for example, have good core strength can lower limb explosive rapid transfer to the upper limb, and help to improve basketball players in the air of the body posture control ability, thus completing the stable and standard shooting movement in the air. Therefore, the core strength training highlights the transfer of strength, coordination, combination and control of muscle characteristics, reflecting the overall, integrated, multi muscle groups in multiple dimensions simultaneously participate in the movement of the new concept. The core strength can improve the working efficiency of the whole body of college athletes, and reduce the unnecessary energy loss in the movement. Have strong core strength as body reserves, body capable of more solid support.

Improve Training Content. In the training process, the core content of special training cannot relax the traditional strength training, through two kinds of training methods in system and complement each other, improve and promote college athletes in the physical confrontation ability and body weight control ability and progress, so as to improve the athletes use the special skill in various competitions and training level at the same time also can be jogging, on students' cardiopulmonary function should be enhanced. At the end of the course, you can learn in accompaniment, gymnastics, stretch to student's body, adjust their mentality, and teachers and students need to complete, when the atmosphere is harmonious, students will tend to be gentle, and get exercise good results, reasonable exercise students' cardiopulmonary function. Arrange sports intensity reasonably. Aerobics in the course of teaching, the main intensity should be moderate, and static, as well as the combination of high and low intensity. The training density should not be too large, the sports load should be arranged reasonably, and the students as the main body should pay attention to the physiological and psychological conditions of the students. Pay attention to rest. The teacher should take the rest of the students and make reasonable arrangements to make sure that they have enough energy during the next practice so that they can devote themselves to the study so as to obtain good teaching results. Normally, students should rest for a few minutes after completing a set of exercises. When the rate of recovery has been increased, the next training session will take place. In the selection of the core strength training, we should select the technical characteristics of sports and the rules of the project, as well as the individual physical conditions of the athletes. In the course of training, we should follow the principle of "first large amount of exercise, low intensity", and take 
static as the main factor. The number of training groups should be increased, and the intermittent questions should be shortened.

Enrich Training Methods. In the process of core strength training of college athletes, different training methods can be adopted according to different positions of players, so as to promote the positive influence of core physical fitness on basketball special position technology. For example, the static balance training, guard by overlooking the bridge of practice, players use side bridge, practice method and center adopts the balance ball single foot support, training methods are different, but the training effect is not different. College physical education is not only to exercise the students' physical quality, but also to improve the other comprehensive qualities of students through various forms. Core strength training can develop self-confidence, imagination, creativity, and analytical problem-solving skills. The introduction of core strength training is the right time for college physical education. Similarly, athletes with different positions for the corresponding technical movements can also use different training methods and methods flexibly. In the course of training special physical ability in colleges and universities, we should pay attention to the combination of special core strength training and traditional strength training, and reasonably arrange the proportion according to the actual situation. In the selection of core strength training methods, we should pay attention to the combination of special technology, and follow the principle of "easy to difficult" and reasonably arrange the intensity of load. In the future training and scientific research experiments, according to the different positions of the basketball players, a more detailed method and means of special core strength training should be worked out. This balance is achieved by providing a relatively stable supporting surface on the instrument or ground. We refer to this state of force training as a steady state of strength training. The unstable state of the body in the course of actual movement destroys the condition of the force that we cultivate in the steady state, and makes it difficult for the force to play in the human movement. This may explain why some athletes show great strength in their equipment strength training, but they cannot perform on the court. Because of the addition of unstable factors in strength training, two major core strength training methods, suspension training and vibration training, have emerged in strength training.

\section{Conclusion}

Traditional strength training is the foundation of the formation of core strength training. The core strength training is a further supplement to the traditional strength training core muscle training. Therefore, in the strength training of college students, we should not only launch the traditional strength training, but also pay more attention to the training of core strength. The strength training in the core area has positive significance to improve the level of sports skills, which is suggested to practice more closely in the future sports training.

\section{References}

[1] Zhao Zhe, Jin Yuqiang, Chang Juan. Research on Core Strength Training for Improving Athletic Ability of Badminton [J]. Journal of Guangzhou Sport University, 2017, 37(3): 81-83.

[2] Kang Ruixin. Research on Trend of Core Strength Training in Basketball Physical Training [J]. China School Physical Education, 2015, 2(5): 74-77.

[3] Liu Yao, Yang Cuimin, Huang Xizhang. Comparative Analysis of Core Strength Training and Traditional Strength Training [J]. China Winter Sports, 2013, 35(2): 58-61.

[4] Wang Ke. Core strength training of college basketball players [J]. Shandong Sports Science \& Technology, 2015, 37(3): 98-101. 